\title{
Spider surgical system versus multiport laparoscopic surgery: performance comparison on a surgical simulator
}

Domenico Giannotti ${ }^{1}$, Giovanni Casella ${ }^{1}$, Gregorio Patrizi ${ }^{*}$, Giorgio Di Rocco ${ }^{1}$, Lidia Castagneto-Gissey ${ }^{1}$, Alessio Metere ${ }^{1}$, Maria Giulia Bernieri², Anna Rita Vestri ${ }^{3}$ and Adriano Redler ${ }^{1}$

\begin{abstract}
Background: The rising interest towards minimally invasive surgery has led to the introduction of laparo-endoscopic single site (LESS) surgery as the natural evolution of conventional multiport laparoscopy. However, this new surgical approach is hampered with peculiar technical difficulties. The SPIDER surgical system has been developed in the attempt to overcome some of these challenges. Our study aimed to compare standard laparoscopy and SPIDER technical performance on a surgical simulator, using standardized tasks from the Fundamentals of Laparoscopic Surgery (FLS).

Methods: Twenty participants were divided into two groups based on their surgical laparoscopic experience: 10 PGY1 residents were included in the inexperienced group and 10 laparoscopists in the experienced group. Participants performed the FLS pegboard transfers task and pattern cutting task on a laparoscopic box trainer. Objective task scores and subjective questionnaire rating scales were used to compare conventional laparoscopy and SPIDER surgical system.

Results: Both groups performed significantly better in the FLS scores on the standard laparoscopic simulator compared to the SPIDER.

Inexperienced group: Task 1 scores (median 252.5 vs. 228.5; $p=0.007$ ); Task 2 scores (median 270.5 vs. 219.0; $p=0.005$ ). Experienced group: Task 1 scores (median 411.5 vs. 309.5; $p=0.005$ ); Task 2 scores (median 418.0 vs. 331.5; $p=0.007$ ).

Same aspects were highlighted for the subjective evaluations, except for the inexperienced surgeons who found both devices equivalent in terms of ease of use only in the peg transfer task.

Conclusions: Even though the SPIDER is an innovative and promising device, our study proved that it is more challenging than conventional laparoscopy in a population with different degrees of surgical experience. We presume that a possible way to overcome such challenges could be the development of tailored training programs through simulation methods. This may represent an effective way to deliver training, achieve mastery and skills and prepare surgeons for their future clinical experience.
\end{abstract}

Keywords: Surgical simulators, Surgical training, Spider, Medical education, Sils, Laparoscopy

\footnotetext{
* Correspondence: g_patrizi@yahoo.com

'Department of Surgical Sciences Policlinico "Umberto I, "Sapienza" University

of Rome, Viale Regina Elena, 324 Rome, Italy

Full list of author information is available at the end of the article
} 


\section{Background}

Since Navarra performed the first single incision laparoscopic cholecystectomy (SILC) in 1997 [1], the rapid advances in minimally invasive surgery have led to the development of several single-port laparoscopic techniques and instruments. The variety of devices and trademarks have spawned a true "battle of acronyms" (SILS, SSLS, SPA, SSL, OPUS, TUES, E-NOTES, NOTUS, etc.), without a definitive consensus name for this new technique of minimally invasive surgery [2].

In 2008 the NOTES Working Group of the Endourological Society and the Laparoendoscopic Single-Site Surgery Consortium for Assessment and Research tried to standardize the terminology to LESS (Laparoendoscopic single site surgery). LESS was defined as any minimally invasive surgical procedure, performed through a single incision/location, using conventional laparoscopic or newly emerging instrumentations. Although the feasibility of LESS has been demonstrated in general, gynecologic, urologic and bariatric surgery, several limitations still affect the single site approach such as lack of instrument triangulation, in-line viewing, cross-handed instrumentation and intra- and extra-corporeal instrument collisions [2]. In the past 5 years several attempts have been made to overcome these drawbacks with the introduction of pre-bent rigid, flexible and articulating laparoscopic instruments. Covidien, Inc. (Norwalk, CT, USA), Novare RealHand HD (Novare Surgical Systems, Cupertino, CA) and Cambridge Endo (Cambridge Endoscopic Devices, Framingham, MA) instruments articulate into the abdomen with different degrees of freedom providing better triangulation. Nevertheless definitive solutions were not provided to avoid cross-handed instrumentations and collisions. Robotic technology is being proposed to reduce the technical challenges of LESS [3]. The da Vinci SingleSite surgical system (Intuitive Surgical, Sunnyvale, CA, USA), the first commercial robot with a kit designed for LESS, allows to overcome the constraint of cross-handed operation by switching the right and left instruments and enables the surgeon's hand to control the instrument on the same side of the screen $[4,5]$. As interest in this new technology continues to grow, a more and more flexible continuum (e.g. IREP robot [6], i-Snake robot [7]) and modular designed robots (e.g. SPRINT robot [8]) are going to be introduced in the surgical market, but outcomes and costs still remain to be defined.

In this context, the research of an ideal single-site surgical platform that might replicate multiport laparoscopy is far from over. In 2009, TransEnterix (Durham, NC, USA) introduced the SPIDER (Single Port Instrument Delivery Extended Reach) surgical system, a single incision surgical device with multiple working channels for rigid and flexible instruments. The system opens up with an umbrella-like system within the abdomen restoring the concept of triangulation without cross-handed instrumentation.

Although the SPIDER has been applied to a variety of surgical procedures [9], there are very few studies evaluating this new surgical platform. Our study aimed to compare standard laparoscopy and SPIDER technical performance on a surgical simulator, using standardized tasks from the Fundamentals of Laparoscopic Surgery (FLS).

\section{Methods}

The study was performed in the Department of Surgical Sciences at "Sapienza"-University of Rome, Rome, Italy. The study was approved by the local Ethics Committee (protocol 518/13) which is the Ethical Committee of the Umberto I Hospital, "Sapienza"-University of Rome. All subjects were enrolled into the study on a voluntary basis and each participant provided full written informed consent. All participants completed a questionnaire assessing demographics as well as number and type of previous laparoscopic procedures. We recruited 20 participants and divided them into two groups according to their experience in laparoscopic surgery. The first group, the inexperienced group, included 10 post-graduate first year residents (PGY 1) in general surgery (mean age 26.1 \pm 1.9 years) with none or low laparoscopic experience (less than 5 laparoscopic procedures, all of them as camera operator). The second group, the LAP experienced group, included 10 surgeons (mean age $36.2 \pm 3.6$ years), performing more than 50 laparoscopic procedures as first operator.

All subjects had no prior experience with FLS and LESS surgery.

Participants performed the FLS pegboard transfer task and the FLS pattern cutting task on a laparoscopic box trainer, using at first conventional laparoscopy for familiarization, then the SPIDER surgical system. To avoid potential outcome inhomogeneity, every participant was blinded to other surgeons' performances.

\section{FLS tasks}

Fundamentals of Laparoscopic Surgery (FLS) is a program of SAGES and the American College of Surgeons (SAGES/ACS, FLS Program, Los Angeles, CA, USA) designed to teach and evaluate the fundamental skills for laparoscopic surgery. The manual skills component is based on the McGill Inanimate System for Training and Evaluation of Laparoscopic Skills (MISTELS) program which consists of five basic tasks performed on a laparoscopic box trainer: pegboard transfer, pattern cutting, endo-loop placement, intracorporeal and extracorporeal knot [10]. In order to evaluate the performance differences between SPIDER and conventional laparoscopy, we chose the pegboard transfer and the pattern cutting 
task. These tasks are not technically challenging and suitable even for beginners. The pegboard transfer task (Task 1) requires the operator to grasp six small pegs from a board, transfer them to the other hand, and place them on a second pegboard. The procedure is then reversed.

As the study included novice participants, the cutoff time was increased from 300 to 600 seconds.

According to MISTELS, the penalty score was defined as the percentage of pegs not transferred as a result of being dropped outside the field of view.

The pattern cutting task (Task 2) requires the participant to cut out a predrawn circle $5 \mathrm{~cm}$ in diameter from a $10 \cdot 10 \mathrm{~cm}^{2}$ piece of gauze suspended between clips. One hand should be used to provide traction on the gauze using the grasper and to place the gauze at more favorable angles for the cutting hand.

As in the first task, the cutoff time was set at $600 \mathrm{sec}-$ onds. According to MISTELS, the penalty was calculated as the percentage of the area of deviation from a perfect circle.

\section{LAP simulator}

The system used in the study was the Simulab LapTrainer (Simulab, Seattle, Washington). It consists of a 22" h, 18" w, 8" d modular plastic box, a boom mounted, fully adjustable 1080p high definition camera with auto-focus, and a Universal Serial Bus (USB) 2.0 card connected to a 17" laptop monitor. Standard-length laparoscopic instruments were inserted through two 12-mm working ports positioned approximately $18 \mathrm{~cm}$ apart in the pliable cover.

\section{SPIDER simulator}

The Simulab LapTrainer was modified to perform FLS tasks using the SPIDER surgical system.

A single $1.8 \mathrm{~cm}$ opening was made on the pliable cover of the box trainer, positioned between the previous port positions. The SPIDER surgical system was inserted through the single opening and was stabilized with an external support arm device.

To perform the FLS task we used a second-generation SPIDER surgical system with a vertebral design of the instrument delivery tubes.

\section{Testing procedure and score calculation}

Before performing tasks, all participants viewed a FLS instructional video illustrating the ideal way to perform each exercise.Testing sessions were conducted on two consecutive days. On the first day, both groups sequentially performed the pegboard transfer (Figure 1a) and the pattern cutting task (Figure 1c) using the LAP simulator. On the second day the same tasks were repeated using the SPIDER simulator (Figure 1b,1d).

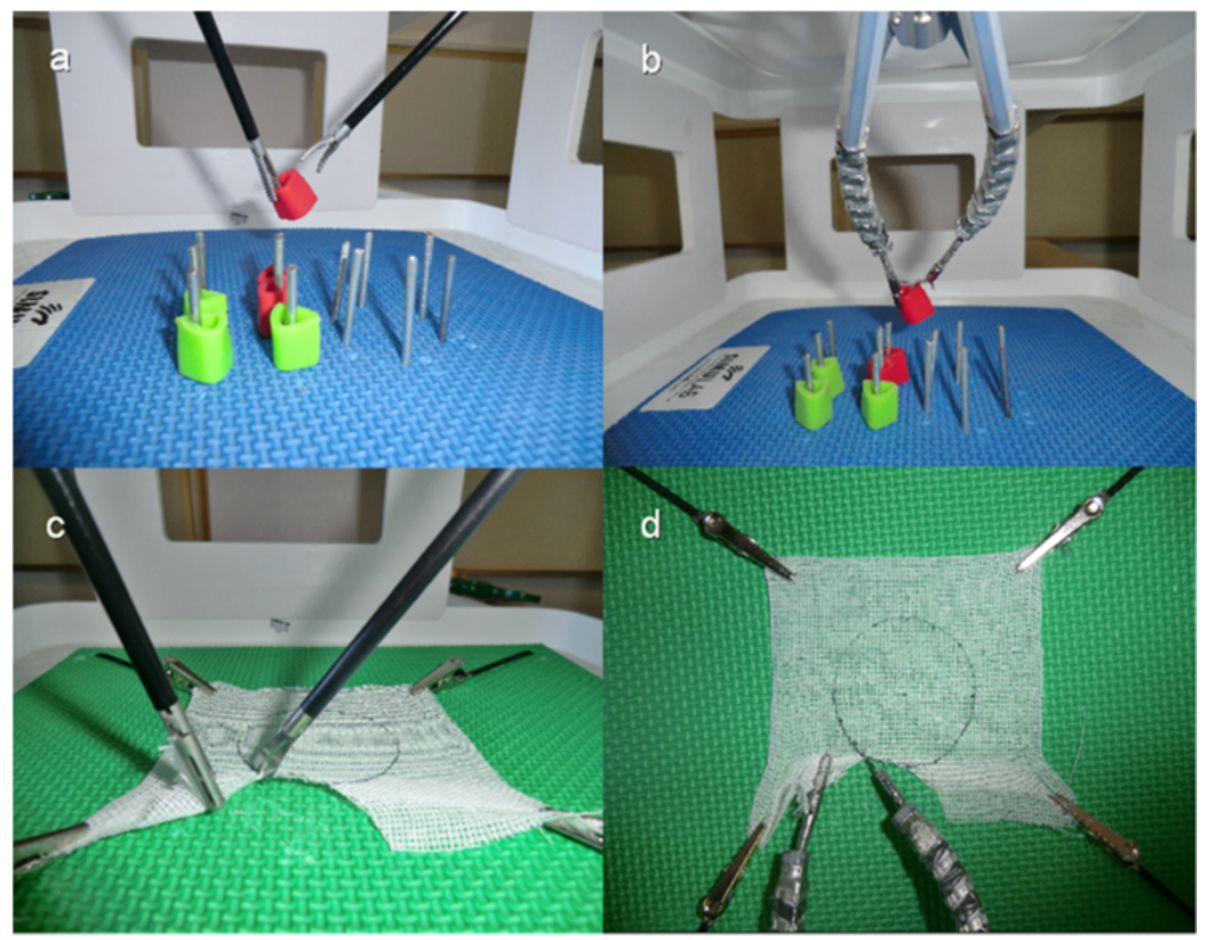

Figure 1 Fundamentals of Laparoscopic Surgery: Conventional laparoscopy versus SPIDER surgical system. a) Peg transfer task on multiport laparoscopic platform; b) Peg transfer task on SPIDER platform; c) pattern cutting task on multiport laparoscopic platform; d) pattern cutting task on SPIDER platform. 
Objective task score and subjective questionnaire rating scales were used to compare conventional laparoscopy and the SPIDER surgical system.

According to established methods of score calculation [8], each task was scored using the following formula in which higher scores reflect better performances:

Task score $=$ cutoff time - completion time - penalty score

Time and penalty measurements were performed by a proctor experienced in FLS evaluation.

A questionnaire was administered to all participants after each task to define conventional laparoscopy and SPIDER's ease of use.

The subjective questionnaire rating was based on a six points Likert scale (1: very difficult, 2: difficult, 3 : somewhat difficult, 4: somewhat easy, 5: easy, 6: very easy).

\section{Statistical analysis}

Continuous variables are presented as mean \pm standard deviation. We assessed the normality of data with the Shapiro-Wilk test. Data of task score and subjective questionnaire ratings do not follow a normal distribution and therefore were reported as median and interquartile range. To evaluate the differences within groups we perform Wilcoxon test. To compare the performance of a specific group on LAP simulator versus SPIDER, we used the Mann-Whitney test and the Median test. A probability value of less than 0.05 was considered as statistically significant. All analyses were carried out with STATA v.12.

\section{Results}

\section{Task score}

Comparing multiport laparoscopy and SPIDER surgical system in the inexperienced group we found significant differences in Task 1 scores (median 252.5 vs. 228.5; $\mathrm{p}=0.007$ ) and in Task 2 scores (median 270.5 vs. 219.0; $\mathrm{p}=0.005)$.

Also the experienced group performed significantly better in Task 1 scores (median 411.5 vs. 309.5; $\mathrm{p}=0.005$ ) and in Task 2 scores (median 418.0 vs. 331.5; $\mathrm{p}=0.007$ ) on the Lap simulator.

When comparing LAP scores between groups, the experienced group had significantly higher scores in both Task 1 (median 411.5 vs. 252.5; $\mathrm{p}=0.002$ ) and Task 2 (median 418.0 vs. 270.5; p=0.005). Likewise, when examining SPIDER scores, the experienced group performed significantly better in both tasks (median 309.5 vs. 228.5; $\mathrm{p}=0.012$ ), (median 331.5 vs. $219.0 ; \mathrm{p}=0.01$ ).

The results of Tasks 1 and 2 are summarized in Tables 1 and 2 respectively.
Table 1 Task 1 (Peg transfer) FLS scores and subjective evaluation of conventional laparoscopy (LAP) vs. SPIDER

\begin{tabular}{|c|c|c|c|c|c|}
\hline \multicolumn{6}{|c|}{ Inexperienced Group } \\
\hline & \multicolumn{2}{|l|}{ LAP } & \multicolumn{2}{|l|}{ SPIDER } & \multirow[b]{2}{*}{ P-value } \\
\hline & Median & IQR & Median & IQR & \\
\hline FLS score & 252.5 & $227.7-347.5$ & 228.5 & $206.0-270.5$ & 0.007 \\
\hline Ease of use & 5.0 & $3.0-4.0$ & 4.0 & $2.7-3.0$ & 0.093 \\
\hline \multicolumn{6}{|c|}{ LAP experienced Group } \\
\hline & \multicolumn{2}{|l|}{ LAP } & \multicolumn{2}{|l|}{ SPIDER } & \multirow[b]{2}{*}{ P-value } \\
\hline & Median & IQR & Median & IQR & \\
\hline FLS score & 411.5 & $399.2-421.2$ & 309.5 & $290.7-338.5$ & 0.005 \\
\hline Ease of use & 5.0 & $5.0-5.2$ & 3.0 & $3.0-4.0$ & 0.004 \\
\hline
\end{tabular}

IQR: interquartile range.

\section{Questionnaire rating scales}

Among the inexperienced group, the ease of use of conventional laparoscopy and SPIDER surgical system did not differ in Task 1 (median 3 vs. 3; $\mathrm{p}=0.093$ ). Task 2 proved to be simpler with conventional laparoscopic instruments as opposed to the SPIDER system (median 3 vs. 2; $\mathrm{p}=0.015$ ).

Furthermore, experienced laparoscopic surgeons found it easier to execute both tasks through conventional laparoscopy rather than with the SPIDER system (Task 1 : median 5 vs. $3 \mathrm{p}=0.004$. Task 2 : median 5 vs. $3 \mathrm{p}=0.002$ ).

Comparing overall personal responses to the questionnaire between groups, on the laparoscopic simulator the experienced group showed significantly higher scores in both Task 1 (median 5 vs. $3 \mathrm{p}=0.002$ ) and Task 2 (median 5 vs. $3 \mathrm{p}=0.0015)$. On the SPIDER simulator no significant differences were found between groups in Task 1 (median 3 vs. $3 \mathrm{p}=0.648$ ) whereas significant differences were seen in Task 2 (median 3 vs. $2 \mathrm{p}=0.0113$ ).

The results of Tasks 1 and 2 are summarized in Tables 1 and 2 respectively.

Table 2 Task 2 (Pattern cutting) FLS scores and subjective evaluation using conventional laparoscopy (LAP) and SPIDER Inexperienced Group

\begin{tabular}{|c|c|c|c|c|c|}
\hline & \multicolumn{2}{|l|}{ LAP } & \multicolumn{2}{|l|}{ SPIDER } & \multirow[b]{2}{*}{ P-value } \\
\hline & Median & IQR & Median & IQR & \\
\hline FLS score & 270.5 & $242.2-347.7$ & 219.0 & $183.7 .-252.5$ & 0.005 \\
\hline Ease of use & 3.0 & $2.7-4.0$ & 2.0 & $2.0-3.0$ & 0.015 \\
\hline \multicolumn{6}{|c|}{ LAP experienced Group } \\
\hline & \multicolumn{2}{|l|}{ LAP } & \multicolumn{2}{|l|}{ SPIDER } & \multirow[b]{2}{*}{ P-value } \\
\hline & Median & IQR & Median & IQR & \\
\hline FLS score & 418.0 & $352.5-432.2$ & 331.5 & $233.0-359.2$ & 0.007 \\
\hline Ease of use & 5.0 & $5.0-5.2$ & 3.0 & $3.0-3.2$ & 0.002 \\
\hline
\end{tabular}

IQR: interquartile range. 


\section{Discussion}

A progressive interest towards minimally invasive procedures has grown in the latest decades leading to the recent introduction of LESS surgery which represents an evolutionary change compared to the classical laparoscopic approach. However, such surgical innovation is associated with peculiar and unique challenges in addition to those already given by conventional laparoscopy [2]. Some of the disadvantages are represented by the lack of triangulation, in-line viewing, cross-handed operating, instrument collisions, crowding of the instrument handles and the ability to target only one or two abdominal quadrants. These ergonomic limitations may transform even simple maneuvers in complicated ones. Even though no ideal instrument still exists, new technologies aim to find a solution to the obstacles preserving its advantages. Articulating instruments can partially restore triangulation also decreasing collisions and in-line viewing.

Although these systems provide better triangulation, the problem of cross-handed instrumentation is yet to be solved. Indeed, without crossing instruments, the surgeons' hands operate in a small field and tend to collide despite the degrees of freedom of the instruments' tips.

The SPIDER surgical system represents an innovative device, introduced in 2009, with the purpose of overcoming several of the limitations mentioned above. It is a disposable single-incision device provided with ergonomic arms and multiple working channels for rigid and flexible instruments. Some of its advantages include the absence of instrument collisions, additional degrees of freedom and an appropriate operative exposure allowing the possibility of triangulation.

Although the SPIDER surgical system has been successfully applied to several surgical procedures including cholecystectomy, nephrectomy and colectomy [9], most studies are referred to case reports or animal models [11-14].

The only human series evaluating the SPIDER platform was retrospectively collected by Gonzalez et al. [15]. These authors compared their first single-incision cholecystectomies performed by standard laparoscopic, robotic and SPIDER platforms with similar results for most of the parameters considered. However, certain selection biases due to differences in mean age and body mass index of patients were identified among the three groups.

Laparoscopic simulators could represent a safe and objective tool for assessing not only surgeon's technical skills [16-22] but also novel surgical instruments [23-25]. Indeed, they can reduce the impact of biases associated to patients' variability by providing a perfectly reproducible and measurable evaluation of the instrument's validity.

Our study evaluated the performance of residents and surgeons with different degrees of experience facing two FLS standardized tasks on a laparoscopic box trainer, using conventional laparoscopy and the SPIDER surgical system. Moreover, questionnaire rating scales were administered to evaluate the ease of use of the surgical devices from a personal point of view.

Overall objective task scores showed that the SPIDER surgical device was more challenging than conventional laparoscopy. As expected, the experienced group performed globally better than the inexperienced one in terms of FLS score on both conventional laparoscopy and SPIDER simulator platforms.

Among the experienced group, significant differences were found between conventional laparoscopy and SPIDER surgical system in Task 1 scores (median 252.5 vs. 228.5; $\mathrm{p}=0.007$ ) and Task 2 scores (median 270.5 vs. 219.0; $\mathrm{p}=0.005$ ). Also in the inexperienced group we found significant differences in Task 1 scores (median 252.5 vs. 228.5; $\mathrm{p}=0.007$ ) and Task 2 scores (median 270.5 vs. $219.0 ; \mathrm{p}=0.005$ ), but the gap in task 1 median scores (median 252.5 vs. 228.5) was smaller compared to the experienced group (median 411.5 vs. 309.5) (Figure 2).

Furthermore, among the inexperienced group, the ease of use of conventional laparoscopy and SPIDER surgical system did not differ in Task 1 (median 3 vs. 3; $\mathrm{p}=0.093$ ) while experienced surgeons found it easier to execute both tasks through conventional laparoscopy rather than with the SPIDER system (Task 1: median 5 vs. $3 \mathrm{p}=0.004$. Task 2: median 5 vs. $3 \mathrm{p}=0.002$ ) (Figure 3).

A possible explanation is that residents were inexperienced with both multiport laparoscopy and SPIDER surgical system while the experienced surgeons were familiar only with multiport laparoscopy. In fact, Lewis et al. demonstrated that previous laparoscopic experience influences the ability to perform simulated tasks with single-incision laparoscopic devices [26].

On the other hand, in Task 2 the SPIDER was more challenging for both groups with significantly lower FLS scores and subjective questionnaire ratings.

This is probably due to the fact that this task requires appropriate traction, exposure of the surgical field and a greater precision when cutting the gauze. Even though the second generation SPIDER is manufactured to increase maneuverability and precision of movement, thanks to the new vertebral design, there is still an undesirable elastic recoil.

Indeed, we were able to ascertain using the SPIDER simulator that it is challenging to gauge with precision the amount of traction and therefore the exposure of the cutting line. At the same time the excessive traction needed has sometimes caused the loss of the instrument's grip with consequent prolonged task accomplishment time.

Furthermore, the SPIDER system provides a lower range of motion compared to conventional laparoscopy especially at the boundaries of the operative field. This problem can be overcome by time-demanding external maneuvers on the supporting arm of the SPIDER itself. 


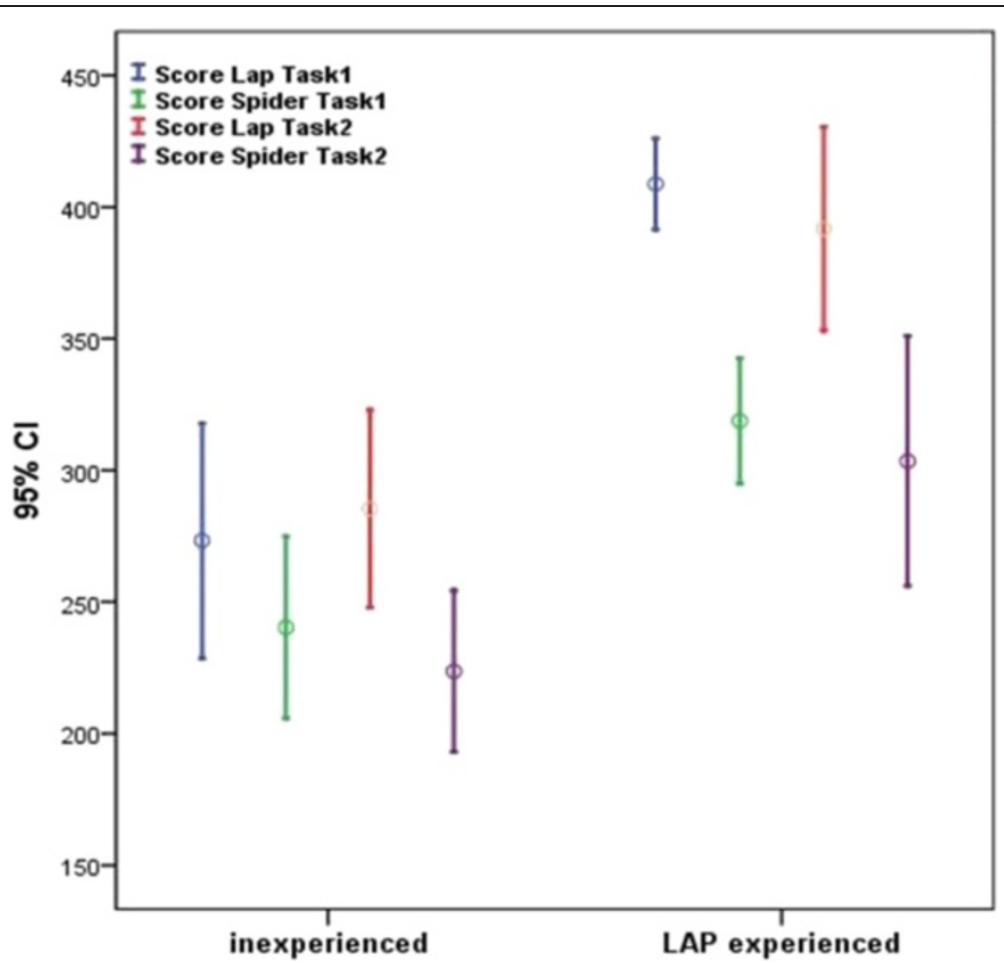

Figure 2 Task 1 and task 2 FLS scores obtained using conventional laparoscopy ( Score Lap) and SPIDER surgical system (Score SPIDER).

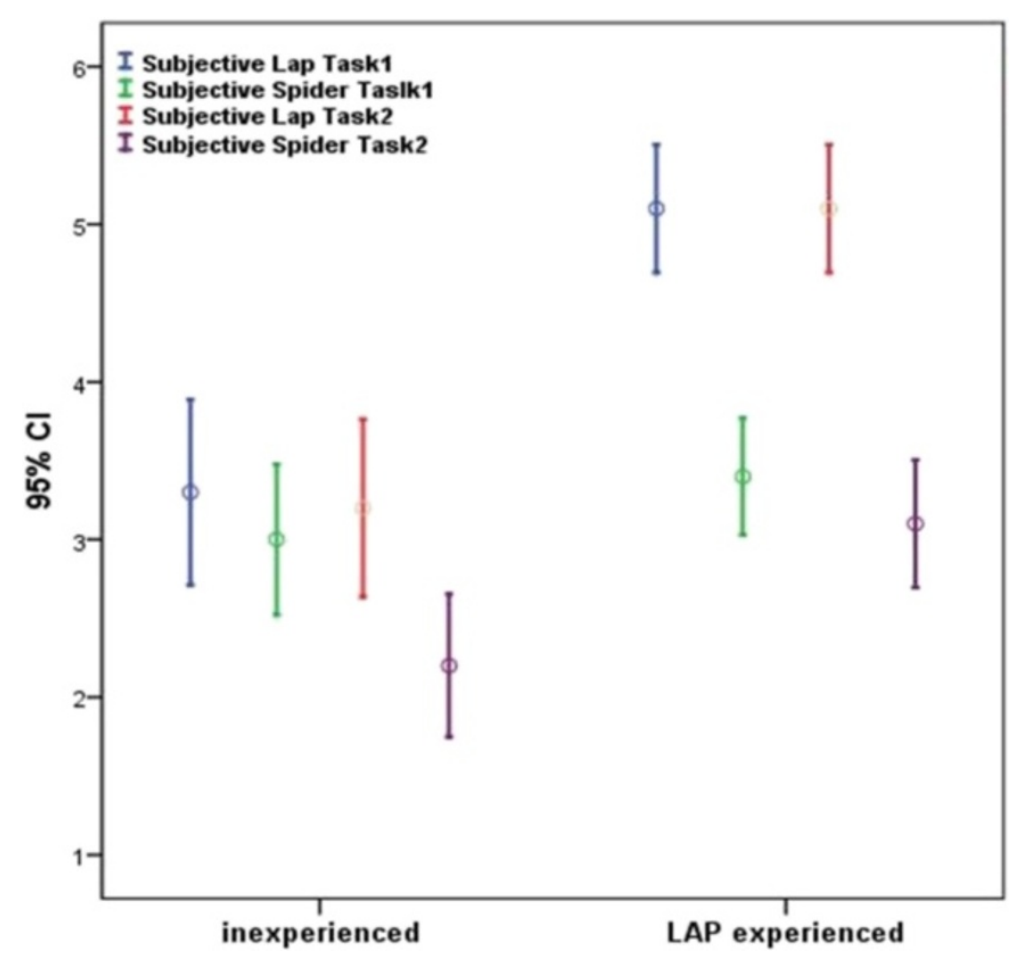

Figure 3 Ease of use of conventional laparoscopy ( Subjective Lap) and SPIDER surgical system (Subjective SPIDER) in task 1 and task 2. 
Our study should be regarded in the context of some limitations. First of all, it might be interesting to perform further studies in order to investigate if a more organized and structured training with both platforms could somehow modify the results. Indeed, the number of reiterations needed to achieve an objective evaluation is strictly dependent on individual experience and therefore extremely hard to define. We already faced this problem in previous studies and, once again, we believe the best option is to perform a single test session to avoid bias [27]. Another limitation was the rather small number of tasks evaluated. We used only two FLS tasks since our aim was to check the performances of inexperienced operators avoiding therefore more challenging tasks as knot tying. Furthermore, since inclusion criteria were no previous experience of FLS and LESS, we reached only ten experienced surgeons who met both criteria. Based on such limitation, we then decided to compare them only with ten novices. This basically represents a convenience sample. Finally, we are planning to perform dedicated studies in the near future in order to compare the SPIDER with other single incision devices on a greater number of participants.

\section{Conclusions}

Fundamental differences exist between multiple-port laparoscopy and single-site incision strategies.

The main advantages of LESS are those of reducing postoperative pain and of improving cosmetics, nonetheless it is also burdened with a number of disadvantages in addition to those already encountered in traditional laparoscopy [2]. A possible way to overcome some of these limitations, unfamiliarity with the instrumentation being one of them, could be the development of tailored training programs through simulation methods. This could possibly represent an effective mean to deliver training, achieve mastery and skills and prepare surgeons for their future clinical experience.

\section{Competing interests}

Drs. Domenico Giannotti, Giovanni Casella, Gregorio Patrizi, Giorgio Di Rocco, Lidia Castagneto-Gissey, Alessio Metere, Maria Giulia Bernieri, Anna Rita Vestri and Adriano Redler have no conflicts of interest or financial ties to disclose.The authors declare that they have no competing interests.

\section{Authors' contributions}

$D G, G P, G C$ designed the study and drafted the manuscript. GDR, LCG, AM collected data and helped drafting the manuscript. ARV, MGB analyzed data. AR critically revised the manuscript. All authors read and approved the final manuscript.

\footnotetext{
Author details

'Department of Surgical Sciences Policlinico "Umberto I, "Sapienza" University of Rome, Viale Regina Elena, 324 Rome, Italy. ${ }^{2}$ Department of Radiology, Oncology and Pathology, "Sapienza" University of Rome, Rome, Italy. ${ }^{3}$ Department of Public Health and Infectious Diseases, "Sapienza" - University of Rome, Rome, Italy.
}

Received: 29 July 2014 Accepted: 21 April 2015

Published online: 03 May 2015

\section{References}

1. Navarra G, Pozza E, Occhionorelli S, Carcoforo P, Donini I. One-wound laparoscopic cholecystectomy. Br J Surg. 1997;84:695.

2. Romanelli JR, Earle DB. Single-port laparoscopic surgery: an overview. Surg Endosc. 2009;23:1419-27.

3. Autorino R, Kaouk JH, Stolzenburg JU, Gill IS, Mottrie A, Tewari A, et al. Current status and future directions of robotic single-site surgery: a systematic review. Eur Urol. 2013;63:266-80.

4. Kroh M, El-Hayek K, Rosenblatt S, Chand B, Escobar P, Kaouk J, et al. First human surgery with a novel single-port robotic system: cholecystectomy using the da Vinci Single-Site platform. Surg Endosc. 2011;25:3566-73.

5. Wren SM, Curet MJ. Single-port robotic cholecystectomy: results from a first human use clinical study of the new da Vinci single-site surgical platform. Arch Surg. 2011;146:1122-7.

6. Ding J, Xu K, Goldman R, Allen P, Fowler D, Simaan N. Design, simulation and evaluation of kinematic alternatives for insertable robotic effectors platforms in single port access surgery. In: Proceedings of the IEEE international conference on robotics and automation (ICRA), May 2010, pp 1053-1058.

7. Shang J, Noonan D, Payne C, Clark J, Sodergren M, Darzi A, et al. An articulated universal joint based flexible access robot for minimally invasive surgery. In: Proceedings of the IEEE international conference on robotics and automation (ICRA), May 2011, pp 1147-1152

8. Petroni G, Niccolini M, Menciassi A, Dario P, Cuschieri A. A novel intracorporeal assembling robotic system for single-port laparoscopic surgery. Surg Endosc. 2013;27:665-70.

9. Villamizar N, Pryor AD. SPIDER and flexible laparoscopy: the next frontier in abdominal surgery. Surg Technol Int. 2010;20:53-8.

10. Fraser SA, Klassen DR, Feldman LS, Ghitulescu GA, Stanbridge D, Fried GM Evaluating laparoscopic skills: setting the pass/fail score for the MISTELS system. Surg Endosc. 2003;17:964-7.

11. Kim SD, Landman J, Sung GT. Laparoendoscopic single-site surgery with the second-generation single port instrument delivery extended reach surgical system in a porcine model. Korean J Urol. 2013;54:327-32.

12. Haber GP, Autorino $R$, Laydner $H$, Yang B, White MA, Hillyer S, et al. SPIDER surgical system for urologic procedures with laparoendoscopic single-site surgery: from initial laboratory experience to first clinical application. Eur Urol. 2012;61:415-22.

13. Salas N, Gorin MA, Gorbatiy V, Castle SM, Bird VG, Leveillee RJ. Laparoendoscopic single site nephrectomy with the SPIDER surgical system: engineering advancements tested in a porcine model. J Endourol. 2011;25:739-42.

14. Pryor AD, Tushar JR, DiBernardo LR. Single-port cholecystectomy with the TransEnterix SPIDER: simple and safe. Surg Endosc. 2010;24:917-23.

15. Gonzalez AM, Rabaza JR, Donkor C, Romero RJ, Kosanovic R, Verdeja JC. Single-incision cholecystectomy: a comparative study of standard laparoscopic, robotic, and SPIDER platforms. Surg Endosc. 2013;27:4524-453.

16. Giannotti D, Patrizi G, Casella G, Di Rocco G, Marchetti M, Frezzotti F, et al. Can virtual reality simulators be a certification tool for bariatric surgeons? Surg Endosc. 2014;28:242-8.

17. Palter VN, Orzech N, Reznick RK, Grantcharov TP. Validation of a structured training and assessment curriculum for technical skill acquisition in minimally invasive surgery: a randomized controlled trial. Ann Surg. 2013;257:224-30.

18. Okrainec A, Soper NJ, Swanstrom LL, Fried GM. Trends and results of the first 5 years of Fundamentals of Laparoscopic Surgery (FLS) certification testing. Surg Endosc. 2011;25:1192-8.

19. Rosenthal ME, Ritter EM, Goova MT, Castellvi AO, Tesfay ST, Pimentel EA, et al. Proficiency-based Fundamentals of Laparoscopic Surgery skills training results in durable performance improvement and a uniform certification pass rate. Surg Endosc. 2010;24:2453-7.

20. Vassiliou MC, Dunkin BJ, Marks JM, Fried GM. FLS and FES: comprehensive models of training and assessment. Surg Clin North Am. 2010;90:535-58.

21. Fried GM. FLS assessment of competency using simulated laparoscopic tasks. J Gastrointest Surg. 2007;12:210-2.

22. Feldman LS, Sherman V, Fried GM. Using simulators to assess laparoscopic competence: ready for widespread use? Surgery. 2004;135:28-42.

23. Conway NE, Romanelli JR, Bush RW, Seymour NE. Ramifications of Single-Port Laparoscopic Surgery: Measuring Differences in Task Performance Using Simulation. Surg Innov. 2014;21:106-11. 
24. Santos BF, Enter D, Soper NJ, Hungness ES. Single-incision laparoscopic surgery $\left(\mathrm{SILS} \mathrm{S}^{\mathrm{TM}}\right)$ versus standard laparoscopic surgery: a comparison of performance using a surgical simulator. Surg Endosc. 2011;25:483-90.

25. Brown-Clerk B, de Laveaga AE, LaGrange CA, Wirth LM, Lowndes BR, Hallbeck MS. Laparoendoscopic single-site (LESS) surgery versus conventional laparoscopic surgery: comparison of surgical port performance in a surgical simulator with novices. Surg Endosc. 2011;25(7):2210-8.

26. Lewis T, Aggarwal R, Kwasnicki R, Darzi A, Paraskeva P. Does previous laparoscopic experience improve ability to perform single-incision laparoscopic surgery? Surg Endosc. 2012;26:1214-9.

27. Giannotti D, Patrizi G, Di Rocco G, Vestri AR, Semproni CP, Fiengo L, et al. Play to become a surgeon: impact of Nintendo Wii training on laparoscopic skills. PLoS One. 2013;8(2), e57372.

\section{Submit your next manuscript to BioMed Central and take full advantage of:}

- Convenient online submission

- Thorough peer review

- No space constraints or color figure charges

- Immediate publication on acceptance

- Inclusion in PubMed, CAS, Scopus and Google Scholar

- Research which is freely available for redistribution 